\title{
Long-term evaluation of home-based pulmonary rehabilitation in patients with COPD
}

\author{
This article was published in the following Dove Press journal: \\ International Journal of COPD \\ 25 September 2015 \\ Number of times this article has been viewed
}

\section{Jean Marie Grosbois ${ }^{1,2}$ \\ Alice Gicquello ${ }^{3}$ \\ Carole Langlois ${ }^{4}$ \\ Olivier Le Rouzic ${ }^{3}$ \\ Frédéric Bart ${ }^{2}$ \\ Benoit Wallaert ${ }^{2,3}$ \\ Cécile Chenivesse ${ }^{5}$}

'FormAction Santé, rue Pietralunga, Pérenchies, ${ }^{2}$ Service de Pneumologie, $\mathrm{CH}$ Béthune, ${ }^{3}$ Service de Pneumologie et Immunoallergologie, Centre des Compétences des Maladies Pulmonaires Rares, Hôpital Calmette, CHRU Lille, ${ }^{4}$ Unité de Biostatistiques, CHRU Lille, ${ }^{5} \mathrm{AP}-\mathrm{HP}$, Groupe Hospitalier Pitié-Salpêtrière Charles Foix, Service de Pneumologie et Réanimation Médicale, Boulevard de l'Hôpital, Paris, France
Correspondence: Jean Marie Grosbois Formaction Santé, rue de Pietralunga, 59840 Pérenchies, France Email jmgrosbois@formactionsante.com
Introduction: Personalized, global pulmonary rehabilitation (PR) management of patients with COPD is effective, regardless of the place in which this rehabilitation is provided. The objective of this retrospective observational study was to study the long-term outcome of exercise capacity and quality of life during management of patients with COPD treated by home-based PR.

Methods: Home-based PR was administered to 211 patients with COPD (mean age, $62.3 \pm 11.1$ years; mean forced expiratory volume in 1 second, $41.5 \% \pm 17.7 \%$ ). Home-based PR was chosen because of the distance of the patient's home from the PR center and the patient's preference. Each patient was individually managed by a team member once a week for 8 weeks with unsupervised continuation of physical exercises on the other days of the week according to an individual action plan. Exercise conditioning, therapeutic patient education, and selfmanagement were included in the PR program. The home assessment comprised evaluation of the patient's exercise capacity by a 6-minute stepper test, Timed Up and Go test, ten times sit-to-stand test, Hospital Anxiety and Depression score, and quality of life (Visual Simplified Respiratory Questionnaire, VQ11, Maugeri Respiratory Failure 28).

Results: No incidents or accidents were observed during the course of home-based PR. The 6-minute stepper test was significantly improved after completion of the program, at 6 months and 12 months, whereas the Timed Up and Go and ten times sit-to-stand test were improved after PR and at 6 months but not at 12 months. Hospital Anxiety and Depression and quality of life scores improved after PR, and this improvement persisted at 6 months and 12 months.

Conclusion: Home-based PR for unselected patients with COPD is effective in the short term, and this effectiveness is maintained in the medium term ( 6 months) and long term (12 months). Home-based PR is an alternative to outpatient management provided all activities, such as exercise conditioning, therapeutic education, and self-management are performed.

Keywords: pulmonary rehabilitation, home, COPD, quality of life, anxiety, depression, selfmanagement

\section{Introduction}

The effectiveness of pulmonary rehabilitation (PR) for patients with COPD has been extensively demonstrated, ${ }^{1-3}$ regardless of the place in which it is performed, in hospital, in an outpatient clinic, or at home. This is true, provided the requirements of this global management program are observed, in combination with exercise conditioning, therapeutic patient education (TPE), and self-management. Outpatient programs are usually proposed, and few studies have been performed on home-based management, ${ }^{4-6}$ although its shortterm effectiveness has been demonstrated in comparison with standard management $t^{4,5}$ or outpatient PR. ${ }^{4,7-9}$ The effectiveness of home-based rehabilitation has also been demonstrated in cystic fibrosis ${ }^{10}$ and in the context of cardiac rehabilitation. ${ }^{11}$

The benefits of outpatient or inpatient PR are not always maintained in the long term (12-24 months).,12 Various long-term physical exercise maintenance programs (monthly 
or weekly sessions, telephone contacts) have demonstrated a significant difference only for exercise capacity at 6 months (but not at 12 months) and no difference in terms of quality of life at 6 months and 12 months. ${ }^{13}$ Some authors ${ }^{14}$ have reported better quality of life at 18 months for the group of patients receiving home-based PR (local rehabilitation structure) compared to patients managed by outpatient PR.

The present study conducted in the Northern part of France reports the results of a retrospective observational routine clinical practice study of home-based rehabilitation management of all patients with COPD referred by their pulmonologist. We formulated the hypothesis that home-based management was safe and effective in terms of exercise capacity, quality of life, and anxiety/depression, and that the results would be maintained at 6 months and 12 months.

\section{Patients and methods}

\section{Patients}

The 8-week home-based PR program was proposed to 226 patients with COPD between January 2010 and December 2013 because of disabling dyspnea experienced during activities of daily living, and out of them, 211 patients performed the PR program (Figure 1). The patient gave his or her written consent and chose home-based management on the basis of personal preference and/or the absence of a local PR center. Exclusion criteria were dementia or poorly controlled psychiatric illness, neurological sequelae, or bone and joint diseases preventing physical activity. Patients with diffuse interstitial lung disease (ILD) or lung cancer currently treated by chemotherapy or radiotherapy and the most severely ill patients who had completed a 6-week home-based program with electrostimulation were not included in this study. Patients receiving oxygen therapy and/or noninvasive ventilation (NIV) and/or with multiple stable comorbidities were able to be included in the rehabilitation program. All data were collected prospectively and were entered into our rehabilitation and TPE-computerized medical records. The Comité d'Evaluation des Protocoles de Recherche Observationnelle (CEPRO) (observational research protocol evaluation committee) of the Société de Pneumologie de Langue Française (French Language Society of Pulmonology) (CEPRO 2011-036) examined this project and approved analysis of the data collected from these patients.

\section{Type of management}

Individual home-based management consisted of 90-minute sessions once a week for 8 weeks, based on an educational needs assessment, and comprising exercise conditioning and resumption of physical activities of daily living, therapeutic

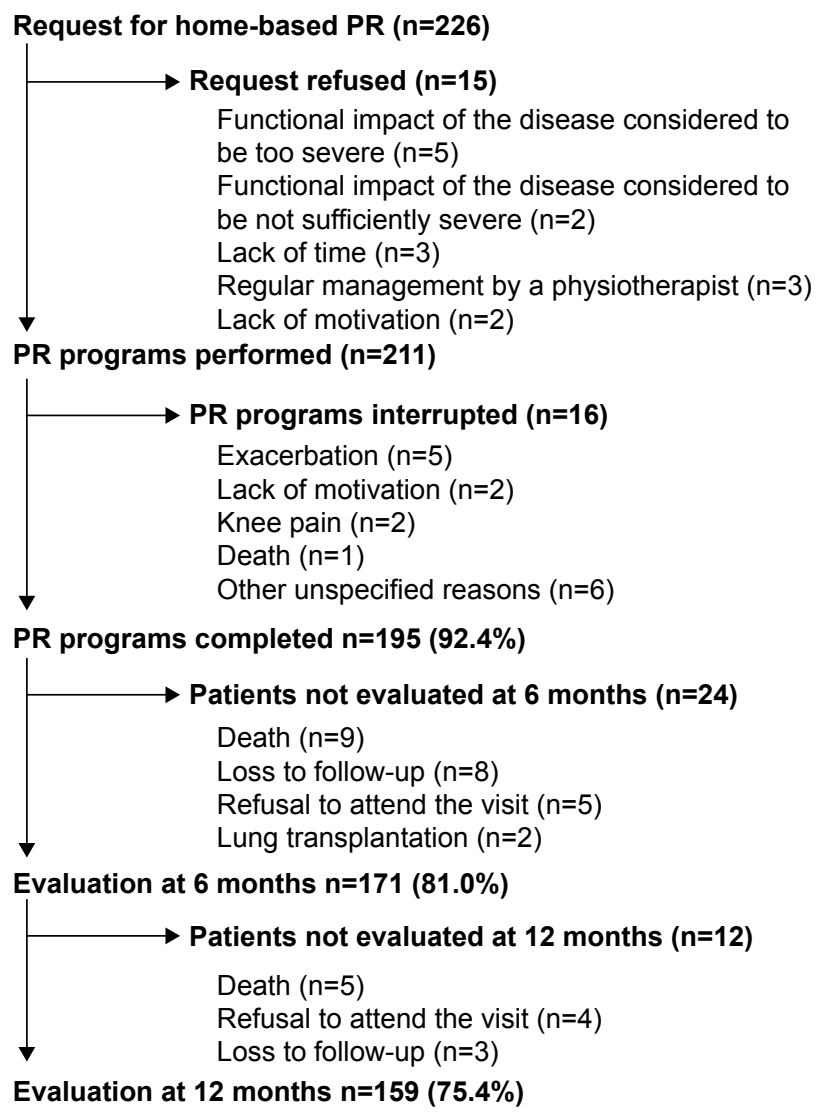

Figure I Flowchart showing participation throughout the study of the patients with COPD managed by home-based PR.

Abbreviation: PR, pulmonary rehabilitation.

patient education, and psychosocial support. Each weekly session was conducted under the direct supervision of a team member (nurses, a sports instructor, a dietician, a medical beautician, and a pulmonologist), while patients continued physical exercises on their own on the other days of the week, according to a personalized action plan. The assessments (exercise capacity, anxiety and depression, and quality of life) were all performed at home before and after the PR program and then again at 6 months and 12 months. Patients were encouraged using motivational interviewings to maintain the lifestyle changes developed during the program on their own and in the long term. Follow-up home visits at 6 months and 12 months were also performed to analyze any difficulties encountered and to showcase improvements and to reinforce motivation, for both the patients and the spouses or caregivers who were frequently presented.

\section{PR program}

\section{Therapeutic education program}

Before starting the rehabilitation program, an educational needs assessment was systematically performed according to the National Health Authority recommendations ${ }^{2}$ at the 
patient's home to evaluate the patient's needs, motivation, and difficulties to any necessary health behavior changes, as well as his/her short-term, medium-term, and long-term life projects to give a real meaning to the PR program. Depending on the patient's needs, the therapeutic education program addressed the following subjects: respiratory diseases and comorbidities (diabetes, cardiovascular diseases, obesity, depression, anxiety), treatments (drugs and inhalor devices), prevention and recognition of exacerbations, physical exercise, outings, sleep, sexuality, breathing management, stress management, balanced diet and weight control, smoking cessation, self-image, self-esteem, etc. This program was individually performed at each visit (usually in the presence of the patient's spouse or caregiver) by interactive presentations, questions and answers, card games, and the use of an illustrated folder. The patient was given patient-validated therapeutic education forms adapted to each session.

\section{Self-management}

Particular attention was paid to the psychological, behavioral, and motivational approach stages of motivation, ${ }^{15,16}$ and stages of acceptance of the disease. Motivational interviewings were designed to help patients increase their intrinsic motivation and, by investigating the ambivalence of some of their harmful health behaviors, help them find solutions to implement favorable health behavior changes. ${ }^{15}$ The objectives of this self-management program ${ }^{17-20}$ were to individually negotiate with each patient the necessary favorable health behavior changes, especially concerning treatment adhesion (drugs and devices), physical exercise, smoking and alcohol cessation, balanced diet, self-esteem, self-image, and stress management.

\section{Exercise conditioning program}

The target conditioning heart rate was determined on a functional exercise test or electrocardiogram (ECG) stress test performed by the patient's referring pulmonologist before inclusion in the program. The exercise conditioning program was conducted under oxygen, with a flow rate adjusted to obtain an $\mathrm{SpO}_{2}>90 \%$ for patients on long-term oxygen therapy (LTOT). Individual exercise bike endurance exercises (Domyos VM 200, Decathlon ${ }^{\circledR}$, Villeneuve-d'Ascq, France) were performed at the target heart rate monitored by a heart rate monitor ([CW Kalenji 100, Decathlon ${ }^{\circledR}$, Villeneuve-d'Ascq, France] which was returned at the fourth week to avoid any medical constraints), with determination of $\mathrm{SpO}_{2}$ by a pulse oximeter (Nonin Oxymontre 3100, Nonin, Plymouth, USA) at each weekly visit. In most cases, this endurance training was initially performed by 10 -minute sequences (or sometimes shorter sequences for the most severely ill patients) optimally adapted to the patient's physical capacities, at least 5 days per week, by trying to achieve 30-45 minutes of exercise, in one or several sessions, during the program. Exercise intensity was progressively adjusted to the target heart rate, and the patient learnt at the same time how to manage the intensity of this exercise depending on the ventilatory threshold, generally corresponding to a score between 4 and 6 (on the Borg 0-10 scale) or a perceived exertion score between 11 and 13 (on the Borg 6-20 scale). A personalized action plan was proposed after discussion with the patient to continue home exercises on the other days of the week. The patient was encouraged to increase the duration of his/her activities of daily living (outings, shopping, walking, home repairs, etc) until the ventilatory threshold, possibly supervised by the rehabilitation professional at the weekly visit. Three daily upper and lower limb muscle strengthening exercises were systematically proposed (with instructions sheets), for 10-15 minutes per day, using weights and dumbbells $(0.5 \mathrm{~kg}$ or $1 \mathrm{~kg})$ and/or Elastiband ${ }^{\mathbb{B}}$ elastic bands. Each exercise comprised a series of ten repeated movements. A 1-minute recovery period was observed between exercises. Finally, warming-up and stretching exercises were also recommended, together with balance exercises whenever necessary. During the program, the patient was encouraged to record on a diary all of the physical activities performed during the week, allowing evaluation and positive reinforcement of the actions. Throughout the program, the team emphasized the need for long-term continuation of physical activities integrated into daily living and endurance training and activities chosen by the patient according to his/ her preferences and local possibilities.

\section{Accident protocol}

The PR agreement handed over to the patient before PR included an accident protocol. An accident was defined as death, hospitalization, or an emergency care requirement for heart or orthopedic disease during the 8 weeks of PR. The patient and/or the therapist could declare accidents occurrence. Patients were asked to interrupt all physical activities in case of any abnormal sensation, especially chest or joint pain and to contact both the rehabilitation team and the attending physician.

\section{Assessment}

Assessment of the patient's lung disease and prescription of treatments, as well as the initial exercise assessment (functional exercise test or stress test), were performed 
by the patient's referring pulmonologist to eliminate any cardiovascular contraindications to exercise conditioning and to define a target heart rate, which corresponded to the heart rate observed at the ventilatory threshold or $50 \%$ of the maximum power observed.

The subsequent assessments of exercise capacity, anxiety, depression, and quality of life were all performed at home, at the beginning and at the end of the PR program and again 6 months and 12 months after completion of the program, by the PR team. The 6-minute stepper test (6MST, Go Sport stepping machine $)^{21-23}$ was used to analyze the number of steps performed in 6 minutes, with the same instructions as those used for the 6-minute walk test (6MWT). Monitoring by a pulse oximeter (Nonin Oxymontre 3100) assessed heart rate and oxygen saturation $\left(\mathrm{SpO}_{2}\right)$ each minute. This test was performed under oxygen in patients receiving LTOT for their lung disease, at the recommended flow rate to obtain an $\mathrm{SpO}_{2}>90 \%$. The Timed Up and Go (TUG), ${ }^{24}$ ten times sit-to-stand test (10STS), ${ }^{25}$ and 6MST were used to measure the exercise capacity. The patient's perceived dyspnea and tiredness of the lower limbs were scored on a Borg 0-10 scale at the end of each of the three tests. ${ }^{26}$

Mood was assessed by the Hospital Anxiety and Depression $(\mathrm{HAD})^{27}$ questionnaire. Quality of life was assessed by means of three questionnaires. The Visual Simplified Respiratory Questionnaire (VSRQ) ${ }^{28}$ comprised eight visual analog scales ranging from 0 to 10 , with a total score ranging from 0 to 80 . A higher score indicated better quality of life. The minimal clinically important difference (MCID) was 3.4 points. $^{28}$ The Maugeri Respiratory Failure 28 (MRF 28) questionnaire, specific to patients with lung disease treated by LTOT and/or NIV, comprised 28 questions ${ }^{29}$ with a total score ranging from $0 \%$ to $100 \%$. A higher score indicated less favorable quality of life. This questionnaire was chosen because of the large population of patients receiving home ventilatory support. The MCID is unknown. The VQ11 comprised 11 items with five levels of response and three components: functional, psychological, and relational (as well as a global component, not included in calculation of the score). ${ }^{30}$ A higher score indicated less favorable quality of life. The MCID is unknown.

Finally, the reasons for withdrawal from the PR program or absence of assessment at 6 months and 12 months were also recorded.

\section{Statistical analysis}

Continuous variables were expressed as mean \pm standard deviation. Qualitative variables were expressed as frequencies or percentages. The normal distribution of continuous variables was tested by the Shapiro-Wilk test. Analysis of variance with repeated measures or a paired Wilcoxon test was used to analyze the time course of the various parameters according to the normal or nonnormal distribution. Post hoc analyses using the Bonferroni procedure were performed. Statistical analysis was performed with SAS 9.3 software (SAS Institute Inc., Cary, NC, USA, NC 25513). A $P$-value $<0.05$ was considered statistically significant.

\section{Results}

The main patient characteristics are summarized in Table 1. The distribution of GOLD stages of obstructive ventilatory disorder was as follows: $26.5 \%$ stage $2,41.2 \%$ stage 3 , $31.3 \%$ stage $4 ; 70.7 \%$ of patients received home ventilatory support (LTOT and/or NIV and/or continuous positive airway pressure); $75.7 \%$ of patients presented three or more comorbidities. The main comorbidities were cardiovascular disease (hypertensive and ischemic heart disease, arrhythmia, and peripheral artery disease), metabolic diseases (obesity, type 2 diabetes, hypothyroidism), rheumatological diseases (osteoporosis, osteoarthritis), and anxiety and depression. Finally, $18.6 \%$ of patients had a BMI $<20 \mathrm{~kg} / \mathrm{m}^{2}$, and $26.2 \%$ of patients had a BMI $>30 \mathrm{~kg} / \mathrm{m}^{2}$.

Table I Baseline characteristics of the 211 patients who performed the PR program

\begin{tabular}{ll}
\hline Age (years) & $62.3 \pm \mathrm{II} . \mathrm{I}$ \\
Male, $\mathrm{n}(\%)$ & $143(67.7)$ \\
$\mathrm{BMI}\left(\mathrm{kg} / \mathrm{m}^{2}\right)$ & $26.8 \pm 7.3$ \\
$\mathrm{FEV}_{1}(\%$ predicted) & $41.5 \pm \mathrm{I} 7.7$ \\
$\mathrm{FEV}_{\mathrm{I}} / \mathrm{FVC}(\%)$ & $50.4 \pm \mathrm{I} 2.8$ \\
Comorbidities, $\mathrm{n}(\%)$ & \\
0 & $3(\mathrm{I} .3)$ \\
$\mathrm{I}$ & $26(\mathrm{II} .5)$ \\
2 & $26(\mathrm{II} .5)$ \\
$\geq 3$ & $17 \mathrm{I}(75.7)$ \\
Treatments, $\mathrm{n}$ (\%) & \\
Short-acting $\beta 2$-agonists & $132(62.5)$ \\
Long-acting $\beta 2$-agonists & $95(45)$ \\
Long-acting anticholinergic & $158(74.9)$ \\
Bronchodilator-corticosteroid combination & $1 \mathrm{I} 4(54)$ \\
Inhaled corticosteroids & $42(19.9)$ \\
Oral corticosteroids & $45(2 \mathrm{I} .3)$ \\
LTOT & $77(36.5)$ \\
LTOT + NIV & $47(22.3)$ \\
LTOT + CPAP & $5(2.4)$ \\
NIV & $8(3.8)$ \\
CPAP & $12(5.7)$ \\
\hline
\end{tabular}

Note: Data are expressed as mean \pm standard deviation.

Abbreviations: $\mathrm{PR}$, pulmonary rehabilitation; $\mathrm{BMI}$, body mass index; $\mathrm{FEV}_{\mathrm{l}}$, mean forced expiratory volume in I second; FVC, forced vital capacity; LTOT, long-term oxygen therapy; NIV, noninvasive ventilation; CPAP, continuous positive airway pressure. 
Table 2 Post hoc analyses comparing baseline, post-PR, 6 months and 12 months variables

\begin{tabular}{|c|c|c|c|c|}
\hline & Baseline & Post-PR & 6 months & 12 months \\
\hline $\mathrm{n}$ & 211 & 195 & $17 \mid$ & 159 \\
\hline 6MST & $328.9 \pm 163$ & $394.7 \pm 172.6 * * *$ & $396.5 \pm 196.6 * * *$ & $432.6 \pm 182.1 * * *$ \\
\hline TUG & $10.4 \pm 5.9$ & $9.2 \pm 7.3 * *$ & $9.6 \pm 5.6^{ \pm}$ & $9.3 \pm 5.4$ \\
\hline IOTSTS & $32.3 \pm 19.1$ & $27.1 \pm 14.3^{* * *}$ & $28.1 \pm 14.3 *$ & $28.6 \pm 14$ \\
\hline HAD & $17.3 \pm 7.3$ & $14.5 \pm 7.3^{* * * *}$ & $13.2 \pm 7.1^{* * * * t \epsilon}$ & $13.9 \pm 7.5^{2 * *, 8}$ \\
\hline MRF 28 & $48.5 \pm 23.4$ & $39.6 \pm 23 * * *$ & $37.3 \pm 23.5 * * *$ & $39.5 \pm 25 * * *, \S$ \\
\hline VQII & $33.4 \pm 9.1$ & $29.7 \pm 9.9 * * *$ & $27.7 \pm 9 * * *, t \epsilon$ & $29.4 \pm 10.3 * * *, \S$ \\
\hline VSRQ & $32.8 \pm 15.5$ & $40.1 \pm 16.4 * * *$ & $41.6 \pm 15.7 * * *$ & $39.6 \pm 16.1 * * *, 5$ \\
\hline
\end{tabular}

Notes: Data are expressed as mean \pm standard deviation. $* P<0.05$, $* * P<0.001$, $* * * P<0.000$ I, statistical comparison with baseline values; ${ }^{t} P<0.05$ and ${ }^{t f} P<0.0$, statistical comparison with post-PR values; ${ }^{\S P}<0.05$, statistical comparison with 6 months values. Data are presented as mean \pm standard.

Abbreviations: PR, pulmonary rehabilitation; 6MST, 6-minute stepper test (number of steps); TUG, Timed Up and Go; IOTSTS, ten times sit-to-stand test; HAD, Hospita Anxiety and Depression questionnaire; MRF 28, Maugeri Respiratory Failure 28; VSRQ, Visual Simplified Respiratory Questionnaire.

Neither accident nor cardiovascular or orthopedic incident due to exercise conditioning was reported during the program.

The results of assessments of exercise capacity (6MST, TUG, 10STS), mood (HAD), and quality of life (VSRQ, MRF 28, VQ11) are summarized in Table 2. A significant improvement was observed for all parameters studied before and after the PR program.

Six months after the program, all these parameters remained significantly improved, except for TUG in comparison with baseline values. At 6 months, the HAD Anxiety score $(P<0.01)$ and the VQ11 score $(P<0.01)$ were significantly improved in comparison with post $P R$ values. There was no significant variation of the other parameters between the values of post PR and 6 months. The 12-month assessment showed persistence of a significant improvement of quality of life, anxiety and depression, and the 6MST in comparison with baseline values. There was no statistically significant difference between post PR and 12 months. The results of the three quality of life questionnaires and the HAD score deteriorated and were statistically significant between 6 months and 12 months $(P<0.05)$. Dyspnea, but not leg tiredness, evaluated on a Borg 0-10 scale at the end of the 6MST (4.7 \pm 1.9$)$, was significantly improved at the three assessments, respectively, $4.2 \pm 1.8(P<0.01)$ after PR, $4.1 \pm 1.8(P<0.01)$ at 6 months, and $4.6 \pm 1.6(P<0.05)$ at 12 months compared with baseline.

\section{Discussion}

This retrospective, observational, clinical trial, conducted in the context of everyday clinical practice in unselected patients with COPD managed by home-based PR, demonstrates that the improvement of exercise capacity, mood, and quality of life observed in the short term was maintained at the 6-month and 12-month assessments.

\section{Safety of home-based PR}

As previously reported, ${ }^{4,5,8}$ the present study confirms that exercise conditioning (exercise bike, walking, climbing stairs, etc) can be safely performed in these patients with COPD with no active cardiovascular disease when the prescription of exercise intensity is based on target heart rate and ventilatory threshold.

\section{Exercise conditioning in home-based exercise PR}

Direct weekly supervision of exercise conditioning at the patient's home improved short-term exercise capacity whether exercise conditioning was performed with an exercise bike $e^{14,31,32}$ or by walking outdoors. ${ }^{7}$ The pragmatic approach of this program integrated exercise bike conditioning, walking, climbing stairs, and, right from the start of the PR program, resumption of physical activities of daily living (housework, home repairs, gardening, outings, shopping, etc) according to a personalized action plan established in collaboration with the patient. Sewell et al showed that the integration of endurance training into the physical activities of daily living during the outpatient PR program was as effective as conventional peripheral upper and lower limb muscle strengthening, ${ }^{33}$ as confirmed by the results of our study. Similarly, review of randomized controlled trials in COPD confirms the efficacy of endurance or resistance training with the use of minimal equipment, a factor that may facilitate home exercises and long-term maintenance of the results. ${ }^{34}$ As patients with severe COPD are known to perform their activities of daily living in the form of short sequences, the objective of PR could be to increase the frequency rather than the duration of these sequences. ${ }^{35}$ Action plans based on daily activities-combined PR lead home-managed patients to integrate exercise into real life, favoring a long-term efficacy of the program. Patients who maintained regular walking at 24 months reported better quality of life and achieved longer walking distances on the 6MWT. ${ }^{36}$ On long-term follow-up, the benefits of PR tended to decrease at 6 months and 12 months, with sometimes better results for quality of life. ${ }^{3}$ A systematic review of seven randomized controlled trials on the effects of supervised exercise conditioning versus usual follow-up revealed a better exercise capacity at 6 months, but not 12 months for supervised patients, with no significant difference in terms of quality of life. ${ }^{13}$ 


\section{Therapeutic patient education and self- management}

The details of educational management are rarely reported in rehabilitation studies. The Living Well With COPD program was used in the study by Maltais et $\mathrm{al}^{8}$ in which TPE was performed on a group basis, in the patient's hospital, at a frequency of two sessions per week for 4 weeks before initiating the home-based exercise conditioning program. The present study demonstrates that an individual approach immediately integrating home-based therapeutic education is feasible and is able to meet the needs of the patient and his/her family in terms of educational objectives and life projects by using adapted tools. However, this approach is only meaningful when it is part of a global approach including self-management ${ }^{19}$ and a motivational approach. ${ }^{15}$ A metaanalysis ${ }^{37}$ has demonstrated the short-term and long-term (1 year) efficacy of motivational interviewing for various health behaviors, with more marked efficacy when interviews are individually and repeatedly performed. Self-management, which comprises medical and behavioral support, life-role support, and emotional support, has been shown to be useful in patients with COPD in terms of improvement of dyspnea and quality of life and decreased hospitalization rates for respiratory problems. ${ }^{18,20,38}$ Active involvement of the spouse and/or caregiver in the very great majority of sessions and the home-based nature of the program, which modifies the patient-carer relationship are probably major determinants in the implementation and follow-up of favorable health behavior changes, ${ }^{39}$ which needs to be studied in more detail in subsequent studies.

\section{Exercise assessment}

The efficacy of this PR program was assessed by means of field evaluation tests performed in the patient's home. The $6 \mathrm{MST}^{21}$ is easy to perform, reproducible, sensitive, and less cumbersome than the 6MWT, which cannot be performed at home. The number of steps on the 6MST is correlated with the distance covered on the 6MWT in patients with chronic respiratory diseases ${ }^{40}$ or ILD. ${ }^{22}$ A previous study shows that improvement of exercise capacity, analyzed by the number of steps on the 6MST before and after program, was identical in the two groups of chronic respiratory insufficiency patients managed by either home-based or outpatient PR. ${ }^{40}$ This confirms that exercise conditioning is effective in both a rehabilitation center or at home provided the prescribed exercise is adapted in terms of intensity (target heart rate or ventilatory threshold), frequency (three to five times per week), and duration (30-45 minutes in one or several sessions). ${ }^{1-3,35}$ This study also shows that the 6MST is a useful field test for the assessment of short-term and long-term exercise capacity. The proposed MCID of 40 steps $^{41}$ was observed at each of the three assessments.

The TUG test has been proposed for the assessment of frail elderly subjects, ${ }^{24}$ and its value for assessment following a cardiac rehabilitation program has been demonstrated. ${ }^{42}$ Various chair stand tests have been validated for use in adults. ${ }^{25}$ The five times sit-to-stand test has been validated in patients with COPD, with a significant improvement after outpatient PR and a MCID of 1.7 seconds. ${ }^{43}$ In the present study, the results of the TUG test and 10STS in this population of patients with COPD were improved at the end of the program, but not at long-term follow-up.

\section{Assessment of mood and quality of life}

Anxiety and depression are frequently present in patients with COPD,${ }^{44}$ where they are associated with a lesser exercise capacity and quality of life. ${ }^{3}$ Improvement in the HAD score is generally observed following PR. ${ }^{1-3,45-48}$ Anxiety and depression worsen dyspnea and reduce performance in COPD. ${ }^{49}$ The long-term positive impact of home-based PR on anxiety and depression might break down the vicious circle between negative emotions, unpleasant respiratory sensations, and poor exercise performance.

Quality of life assessment is an essential component of PR programs. The most commonly used quality of life questionnaires specific to respiratory diseases are $\mathrm{CRQ}^{50}$ and SGRQ. ${ }^{51}$ However, these questionnaires are too long to be applied in routine clinical practice. Shorter questionnaires have been developed, including COPD Assessment Test, ${ }^{52}$ VSRQ, ${ }^{28}$ and VQ11. ${ }^{30}$ The present study demonstrated a significant improvement, over a 1-year horizon, of quality of life as assessed by means of a total VSRQ score higher than the MCID of 3.4 points and the three dimensions of the VQ11 (functional, psychological, relational). Interestingly, the MRF 28 was also improved in this population of patients with severe COPD, $70.7 \%$ of whom were treated by NIV and/or LTOT.

\section{Limitations}

This was a retrospective, observational, routine clinical practice study. Patients were not randomized, and the decision to perform home-based PR was dependent on the patient's personal preferences and/or the distance between the patient's home and the PR center. However, this situation corresponds to the problems encountered in everyday practice by physicians wishing to prescribe and patients wishing to enter in a 
$\mathrm{PR}$ program. Home-based $\mathrm{PR}$ is one of the possible responses proposed to pulmonologists (and general physicians) wishing to prescribe this type of management to their patients. Homebased management meets the needs of certain patients for various reasons: difficulties getting around, lack of motivation to attend group rehabilitation and/or attend an inpatient or outpatient center, transport problems, distance from the nearest PR center, disruption of everyday habits, duration of the program, etc. ${ }^{3,5,8,53,54}$

\section{Conclusion}

In conclusion, home-based PR for patients with COPD is effective in terms of exercise capacity, anxiety and depression, and quality of life in short term, medium term (6 months), and long term (12 months). Home-based PR constitutes an alternative to outpatient or inpatient rehabilitation and may facilitate the development and improve the accessibility of PR to a greater number of patients all over the country. In addition to exercise conditioning, TPE and self-management (behavioral approach, motivational interviewing, acceptance of the disease), which correspond not only to the definition of PR, but also to the assessment of exercise capacity by means of field tests and short quality of life questionnaires, can now be fully performed at home by a motivated cross-disciplinary team trained in this form of global management.

\section{Acknowledgments}

The authors thank the rehabilitation team who managed these patients: GT, SD, FU, VW, and ML. The authors also thank Adair, France Oxygène, Homeperf, LVL, Orkyn, Santélys, SOS Oxygène, Sysmed, VitalAire, and the ARS Nord Pas De Calais for their support of the home-based PR program.

\section{Disclosure}

The authors report no conflicts of interest in this work.

\section{References}

1. Lacasse Y, Goldstein R, Lasserson TJ, Martin S. Pulmonary rehabilitation for chronic obstructive pulmonary disease. Cochrane Database Syst Rev. 2006;(4):CD003793.

2. Société de Pneumologie de Langue Française. Recommandations pour la pratique clinique. Prise en charge de la BPCO. Rev Mal Respir. 2010; 27(suppl 1):S1-S76.

3. Spruit MA, Singh SJ, Garvey C, et al; ATS/ERS Task Force on Pulmonary Rehabilitation. An official American Thoracic Society/ European Respiratory Society statement: key concepts and advances in pulmonary rehabilitation. Am J Respir Crit Care Med. 2013;188(8): e13-e64.

4. Vieira DSR, Maltais F, Bourbeau J. Home-based pulmonary rehabilitation in chronic obstructive pulmonary disease patients. Curr Opin Pulm Med. 2010;16(2):134-143.
5. Liu XL, Tan JY, Wang T, et al. Effectiveness of home-based pulmonary rehabilitation for patients with chronic obstructive pulmonary disease: a meta-analysis of randomized controlled trials. Rehabil Nurs. 2014; 39(1):36-59.

6. McGavin CR, Gupta SP, Lloyd EL, McHardy GJ. Physical rehabilitation for the chronic bronchitic: results of a controlled trial of exercises in the home. Thorax. 1977;32(3):307-311.

7. Puente-Maestu L, Sánz ML, Sánz P, Cubillo JM, Mayol J, Casaburi R. Comparison of effects of supervised versus self-monitored training programmes in patients with chronic obstructive pulmonary disease. Eur Respir J. 2000;15(3):517-525.

8. Maltais F, Bourbeau J, Shapiro S, et al; Chronic Obstructive Pulmonary Disease Axis of Respiratory Health Network, Fonds de recherche en santé du Québec. Effects of home-based pulmonary rehabilitation in patients with chronic obstructive pulmonary disease: a randomized trial. Ann Intern Med. 2008;149(12):869-878.

9. Güell MR, de Lucas P, Gáldiz JB, et al. Comparación de un programa de rehabilitación domiciliario con uno hospitalario en pacientes con EPOC: estudio multicéntrico español. Arch Bronconeumol. 2008; 44(10):512-518.

10. Hebestreit H, Kieser S, Junge S, et al. Long-term effects of a partially supervised conditioning programme in cystic fibrosis. Eur Respir J. 2010;35(3):578-583.

11. Taylor RS, Dalal H, Jolly K, Moxham T, Zawada A. Home-based versus centre-based cardiac rehabilitation. Cochrane Database Syst Rev. 2010;(1):CD007130.

12. Ochmann U, Jörres RA, Nowak D. Long-term efficacy of pulmonary rehabilitation: a state-of-the-art review. J Cardiopulm Rehabil Prev. 2012;32(3):117-126.

13. Beauchamp MK, Evans R, Janaudis-Ferreira T, Goldstein RS, Brooks D. Systematic review of supervised exercise programs after pulmonary rehabilitation in individuals with COPD. Chest. 2013;144(4): 1124-1133.

14. Strijbos JH, Postma DS, van Altena R, Gimeno F, Koëter GH. A comparison between an outpatient hospital-based pulmonary rehabilitation program and a home-care pulmonary rehabilitation program in patients with COPD. A follow-up of 18 months. Chest. 1996;109(2):366-372.

15. Miller WR, Rollnick S. Motivational Interviewing: Preparing People for Change. 2nd ed. New York: Guilford Press; 2002.

16. Kristeller JL, Rossi JS, Ockene JK, Goldberg R, Prochaska JO. Processes of change in smoking cessation: a cross-validation study in cardiac patients. J Subst Abuse. 1992;4(3):263-276.

17. Lacroix A, Assal J-P. L'éducation Thérapeutique Des PatientsAccompagner Les Patients Avec Une Maladie Chronique: Nouvelles Approches. 3ème ed. Paris: Maloine; 2011.

18. Bourbeau J, van der Palen J. Promoting effective self-management programmes to improve COPD. Eur Respir J. 2009;33(3):461-463.

19. Bourbeau J, Nault D, Dang-Tan T. Self-management and behaviour modification in COPD. Patient Educ Couns. 2004;52(3):271-277.

20. Zwerink M, Brusse-Keizer M, van der Valk PD, et al. Self management for patients with chronic obstructive pulmonary disease. Cochrane Database Syst Rev. 2014;3:CD002990.

21. Borel B, Fabre C, Saison S, Bart F, Grosbois J-M. An original field evaluation test for chronic obstructive pulmonary disease population: the six-minute stepper test. Clin Rehabil. 2010;24(1):82-93.

22. Delourme J, Stervinou-Wemeau L, Salleron J, Grosbois JM, Wallaert B. Six-minute stepper test to assess effort intolerance in interstitial lung diseases. Sarcoidosis Vasc Diffuse Lung Dis. 2012;29(2):107-112.

23. Coquart JB, Lemaître F, Castres I, Saison S, Bart F, Grosbois J-M. Reproducibility and sensitivity of the 6-minute stepper test in patients with COPD. COPD. Epub 2015 Aug 19.

24. Podsiadlo D, Richardson S. The timed "Up \& Go": a test of basic functional mobility for frail elderly persons. J Am Geriatr Soc. 1991;39(2): $142-148$.

25. Netz Y, Ayalon M, Dunsky A, Alexander N. "The multiple-sit-to-stand" field test for older adults: what does it measure? Gerontology. 2004; 50(3):121-126. 
26. Borg GA. Psychophysical bases of perceived exertion. Med Sci Sports Exerc. 1982;14(5):377-381.

27. Lepine JP, Godchau M, Brun P. Anxiety and depression in inpatients. Lancet. 1985;2(8469-8470):1425-1426.

28. Perez T, Arnould B, Grosbois JM, et al; TIPHON Study Group. Validity, reliability, and responsiveness of a new short Visual Simplified Respiratory Questionnaire (VSRQ) for health-related quality of life assessment in chronic obstructive pulmonary disease. Int J Chron Obstruct Pulmon Dis. 2009;4:9-18.

29. Janssens JP, Héritier-Praz A, Carone M, et al. Validity and reliability of a French version of the MRF-28 health-related quality of life questionnaire. Respiration. 2004;71(6):567-574.

30. Ninot G, Soyez F, Fiocco S, Nassih K, Morin AJS, Prefaut C. Le VQ11, un questionnaire de qualité de vie spécifique à la $\mathrm{BPCO}$ utilisable en clinique. Rev Mal Respir. 2010;27(5):472-481.

31. Debigaré R, Maltais F, Whittom F, Deslauriers J, LeBlanc P. Feasibility and efficacy of home exercise training before lung volume reduction. J Cardiopulm Rehabil. 1999;19(4):235-241.

32. Wijkstra PJ, van der Mark TW, Kraan J, van Altena R, Koëter GH, Postma DS. Effects of home rehabilitation on physical performance in patients with chronic obstructive pulmonary disease (COPD). Eur Respir J. 1996;9(1):104-110.

33. Sewell L, Singh SJ, Williams JEA, Collier R, Morgan MDL. Can individualized rehabilitation improve functional independence in elderly patients with COPD? Chest. 2005;128(3):1194-1200.

34. Alison JA, McKeough ZJ. Pulmonary rehabilitation for COPD: are programs with minimal exercise equipment effective? J Thorac Dis. 2014;6(11):1606-1614

35. Hill K, Vogiatzis I, Burtin C. The importance of components of pulmonary rehabilitation, other than exercise training, in COPD. Eur Respir Rev. 2013;22(129):405-413.

36. Heppner PS, Morgan C, Kaplan RM, Ries AL. Regular walking and long-term maintenance of outcomes after pulmonary rehabilitation. J Cardiopulm Rehabil. 2006;26(1):44-53.

37. Lundahl B, Burke BL. The effectiveness and applicability of motivational interviewing: a practice-friendly review of four meta-analyses. J Clin Psychol. 2009;65(11):1232-1245.

38. Benzo R, Vickers K, Ernst D, Tucker S, McEvoy C, Lorig K. Development and feasibility of a self-management intervention for chronic obstructive pulmonary disease delivered with motivational interviewing strategies. J Cardiopulm Rehabil Prev. 2013;33(2):113-123.

39. Kaptein AA, Fischer MJ, Scharloo M. Self-management in patients with COPD: theoretical context, content, outcomes, and integration into clinical care. Int J Chron Obstruct Pulmon Dis. 2014;9:907-917.

40. Grosbois J-M, Le Rouzic O, Monge E, Bart F, Wallaert B. La réhabilitation respiratoire: évaluation de deux types de prise en charge, ambulatoire versus domicile. Rev Pneumol Clin. 2013;69(1):10-17.
41. Pichon R, Beaumont M, Le Ber-Moy C, Péran L, Couturaud F. Détermination d'une différence minimale importante pour le steppertest de six minutes chez les patients atteints de BPCO. Rev Mal Respir. 2015;32:A75.

42. Bellet RN, Francis RL, Jacob JS, et al. Timed Up and Go Tests in cardiac rehabilitation: reliability and comparison with the 6-Minute Walk Test. J Cardiopulm Rehabil Prev. 2013;33(2):99-105.

43. Jones SE, Kon SS, Canavan JL, et al. The five-repetition sit-to-stand test as a functional outcome measure in COPD. Thorax. 2013;68(11): $1015-1020$

44. Jones PW, Baveystock CM, Littlejohns P. Relationships between general health measured with the sickness impact profile and respiratory symptoms, physiological measures, and mood in patients with chronic airflow limitation. Am Rev Respir Dis. 1989;140(6):1538-1543.

45. Withers NJ, Rudkin ST, White RJ. Anxiety and depression in severe chronic obstructive pulmonary disease: the effects of pulmonary rehabilitation. J Cardiopulm Rehabil. 1999;19(6):362-365.

46. Griffiths TL, Burr ML, Campbell IA, et al. Results at 1 year of outpatient multidisciplinary pulmonary rehabilitation: a randomised controlled trial. Lancet. 2000;355(9201):362-368.

47. Coventry PA. Does pulmonary rehabilitation reduce anxiety and depression in chronic obstructive pulmonary disease? Curr Opin Pulm Med. 2009;15(2):143-149.

48. Harrison SL, Greening NJ, Williams JEA, Morgan MDL, Steiner MC, Singh SJ. Have we underestimated the efficacy of pulmonary rehabilitation in improving mood? Respir Med. 2012;106(6):838-844.

49. von Leupoldt A, Taube K, Lehmann K, Fritzsche A, Magnussen H. The impact of anxiety and depression on outcomes of pulmonary rehabilitation in patients wwith COPD. Chest. 2011;140(3):730-736.

50. Guyatt GH, Berman LB, Townsend M, Pugsley SO, Chambers LW. A measure of quality of life for clinical trials in chronic lung disease. Thorax. 1987;42(10):773-778.

51. Jones PW, Quirk FH, Baveystock CM, Littlejohns P. A self-complete measure of health status for chronic airflow limitation. The St George's Respiratory Questionnaire. Am Rev Respir Dis. 1992;145(6): 1321-1327.

52. Jones PW, Harding G, Berry P, Wiklund I, Chen W-H, Kline Leidy N. Development and first validation of the COPD assessment test. Eur Respir J. 2009;34(3):648-654.

53. Keating A, Lee A, Holland AE. What prevents people with chronic obstructive pulmonary disease from attending pulmonary rehabilitation? A systematic review. Chron Respir Dis. 2011;8(2):89-99.

54. Hayton C, Clark A, Olive S, et al. Barriers to pulmonary rehabilitation: characteristics that predict patient attendance and adherence. Respir Med. 2013;107(3):401-407.
International Journal of COPD

\section{Publish your work in this journal}

The International Journal of COPD is an international, peer-reviewed journal of therapeutics and pharmacology focusing on concise rapid reporting of clinical studies and reviews in COPD. Special focus is given to the pathophysiological processes underlying the disease, intervention programs, patient focused education, and self management protocols.
Dovepress

This journal is indexed on PubMed Central, MedLine and CAS. The manuscript management system is completely online and includes a very quick and fair peer-review system, which is all easy to use. Visit http://www.dovepress.com/testimonials.php to read real quotes from published authors. 